\title{
The feasibility and safety of high-intensity focused ultrasound combined with low-dose external beam radiotherapy as supplemental therapy for advanced prostate cancer following hormonal therapy
}

\author{
Rui-Yi Wu ${ }^{1}$, Guo-Min Wang ${ }^{1}$, Lei Xu ${ }^{1}$, Bo-Heng Zhang ${ }^{2}$, Ye-Qing Xu' ${ }^{1}$, Zhao-Chong Zeng ${ }^{3}$ and Bing Chen ${ }^{3}$
}

The aim of this study was to investigate the feasibility and safety of high-intensity focused ultrasound (HIFU) combined with (+) low-dose external beam radiotherapy (LRT) as supplemental therapy for advanced prostate cancer (PCa) following hormonal therapy (HT). Our definition of HIFU+LRT refers to treating primary tumour lesions with HIFU in place of reduced field boost irradiation to the prostate, while retaining four-field box irradiation to the pelvis in conventional-dose external beam radiotherapy (CRT). We performed a prospective, controlled and non-randomized study on 120 patients with advanced PCa after HT who received HIFU, CRT, HIFU+LRT and HT alone, respectively. CT/MR imaging showed the primary tumours and pelvic lymph node metastases visibly shrank or even disappeared after HIFU+LRT treatment. There were significant differences among four groups with regard to overall survival (OS) and disease-specific survival (DSS) curves ( $P=0.018$ and 0.015$)$. Further comparison between each pair of groups suggested that the long-term DSS of the HIFU+LRT group was higher than those of the other three groups, but there was no significant difference between the HIFU+LRT group and the CRT group. Multivariable Cox's proportional hazard model showed that both HIFU+LRT and CRT were independently associated with DSS $(P=0.001$ and 0.035$)$ and had protective effects with regard to the risk of death. Compared with CRT, HIFU+LRT significantly decreased incidences of radiation-related late gastrointestinal (GI) and genitourinary (GU) toxicity grade $\geqslant$ II. In conclusion, long-term survival of patients with advanced PCa benefited from strengthening local control of primary tumour and regional lymph node metastases after HT. As an alternative to CRT, HIFU+LRT showed good efficacy and better safety.

Asian Journal of Andrology (2011) 13,499-504; doi:10.1038/aja.2010.153; published online 14 March 2011

Keywords: complication; high-intensity focused ultrasound; hormonal therapy; low-dose external beam radiotherapy; prostate cancer; survival rate

\section{INTRODUCTION}

In recent years, the incidence of prostate cancer (PCa) in Asia, including China, has shown a clear upward trend. ${ }^{1}$ As PCa occurs insidiously, the majority of patients visiting the clinic have progressed to locally advanced $\left(\mathrm{T}_{3-4}\right)$ or metastatic disease. At present, androgen-ablative hormonal therapy is the mainstay for the management of advanced PCa. However, most patients with locally advanced disease and almost all patients with distant metastases go on to develop hormone-refractory $\mathrm{PCa}$, which results in the loss of sensitivity to antiandrogen and chemotherapeutic drugs and is associated with a poor prognosis. ${ }^{2,3}$ Adjuvant radiotherapy can improve the therapeutic effects with respect to locally advanced $\mathrm{PCa}$, but the radiation to the prostate is inevitably delivered to surrounding normal organs. This off-target radiation often induces severe late gastrointestinal (GI) and genitourinary (GU) complications that affect the patient's quality of life. ${ }^{4,5}$ Low-dose radiation therapy involves decreased radiationrelated toxicity, which also impairs its therapeutic effect. Utilizing new minimally invasive therapies to replace or modify the traditional radiotherapy procedure is a feasible approach to reduce the dose of radiation.

High-intensity focused ultrasound (HIFU) is a new minimally invasive energy-ablation technique for solid tumours. High-frequency ultrasound waves generated by an extracorporeal transducer are directionally focused on the target within the body (i.e., tumour site). The thermal effects (focus temperature: $70-100^{\circ} \mathrm{C}$ ) and non-thermal effects (mechanical effects, acoustic cavitation, etc.) can cause coagulative necrosis or the collapse of tumour tissue. As each HIFU exposure treats a precisely defined portion of the targeted tissue under positioning and real-time monitoring of ultrasound or MRI, its 'ablation' margin is similar to the surgical margin, without damage to the surrounding normal tissues. ${ }^{6}$ In addition, the synergistic effect of HIFU and radiotherapy has been confirmed. ${ }^{7}$ However, for advanced PCa, the invasive margin of the tumour and pelvic lymph nodes are the blind zone beyond the target areas of HIFU treatment.

${ }^{1}$ Department of Urology, Zhongshan Hospital, Fudan University, Shanghai 200032, China; ${ }^{2}$ Evidence-Based Medicine Center, Fudan University, Shanghai 200032, China and ${ }^{3}$ Department of Radiotherapy, Zhongshan Hospital, Fudan University, Shanghai 200032, China

Correspondence: Professor GM Wang (wang.guomin@zs-hospital.sh.cn)

Received: 24 May 2010; Revised: 2 July 2010; Accepted: 14 October 2010; Published online: 14 March 2011 
Therefore, we sought to treat primary tumour lesions with HIFU in place of reduced field boost irradiation to the prostate, while retaining four-field box irradiation to the pelvis in conventional-dose external beam radiotherapy (CRT). The reliable local effects of HIFU assured local control of the prostatic primary tumour, while radiotherapy not only covered the blind zone, but also had a synergistic effect in combination with HIFU on the primary tumour. At the same time, the combined treatment protocols decreased the total irradiation dose, especially with regard to the influence of radiation on the GI and GU tract around the prostate, which protected patients from severe late radiation-related complications. Between March 2000 and March 2010, we performed a prospective, controlled and non-randomized study on 120 patients with advanced PCa after hormonal therapy (HT) and investigated the efficacy and safety of HIFU+low-dose external beam radiotherapy (LRT) as supplemental therapy for advanced PCa.

\section{MATERIALS AND METHODS}

\section{Patients}

Between March 2000 and March 2010, 120 patients were admitted to Zhongshan Hospital of Fudan University, including locally advanced PCa $\left(\mathrm{T}_{3-4} \mathrm{~N}_{0-1} \mathrm{M}_{0}\right)$ and metastatic PCa (any $\mathrm{T}$, any $\mathrm{N}$ and $\left.\mathrm{M}_{1}\right)$. All study participants were first-time patients who presented with adenocarcinomas. Each showed a clear prostate-specific antigen (PSA) response after primary hormone therapy. HT introduced surgical castration combined with non-steroidal anti-androgens in accordance with the principles of intermittent maximal androgen blockade. All patients took non-steroidal anti-androgens continuously, which were then withdrawn after about 6-9 months' HT induction with PSA reduction $<4 \mathrm{ng} \mathrm{ml}^{-1}$. Patients started a second cycle of treatment when PSA values rose $>20 \mathrm{ng} \mathrm{ml}^{-1}$. After about 3 months of HT, patients were divided into four groups according to their toleration of treatment: HIFU+LRT, HIFU, CRT and HT alone (i.e., control group). All patients were informed about the available treatment options and then elected to undergo treatment and signed the informed consent form. There were no significant differences among the four groups with respect to age, initial PSA, Gleason score or clinical stage (Table 1).

\section{HIFU therapy}

The FEP-BY HIFU therapy system (Yuande Bio-Medical Engineering Co., Ltd, Beijing, China) was used for extracorporeal HIFU therapy (Figure 1), as previously described in detail, ${ }^{8}$ and was approved by the State Food and Drug Administration of China. The therapy system comprises HIFU transducer, ultrasound imaging system, treatment table and seat frames, water degassing system and computer control station. The system is equipped with two (upper and lower) transducers, which facilitate the treatment of tumours in different parts of the body. Each transducer has a concave spherical surface with a sparse array of ceramic piezoelectric ultrasound emitters that converge from a wide angle to focus on a designated focal point inside the body. The US frequencies used for treatment were $1.04 \mathrm{MHz}$. The size of the cigar-shaped focus was $3 \times 3 \times 8 \mathrm{~mm}$.

Patients took laxatives to empty their bowels the night before treatment. Through the inserted urethral catheter, the bladder was filled with degassed water to facilitate prostate targeting before HIFU therapy. The wide convergence angle $\left(80^{\circ}\right)$ of the high-intensity ultrasound beam maintained the acoustic intensity at the skin surface below the threshold of skin pain and burn; therefore, no anaesthesia was needed. Patients sat on the seat frames and remained seated. The lower HIFU transducer was used to treat PCa, and warmed degassed
Table 1 Baseline demographic and clinical characteristics

\begin{tabular}{|c|c|c|c|c|c|}
\hline & $\begin{array}{c}H I F U+L R T \\
(\mathrm{n}=30)\end{array}$ & $\begin{array}{c}\text { HIFU } \\
(\mathrm{n}=30)\end{array}$ & $\begin{array}{c}C R T \\
(\mathrm{n}=30)\end{array}$ & $\begin{array}{l}\text { Control } \\
(n=30)\end{array}$ & $\mathrm{P}$ value \\
\hline Age (years) & $69(66-74)$ & 72 (69-74) & 72.5 (67-77) & 72 (69-76) & $0.088 *$ \\
\hline $\begin{array}{l}\text { Initial PSA } \\
\quad\left(\mathrm{ngml}^{-1}\right)\end{array}$ & $\begin{array}{c}91.9(59.6- \\
114.3)\end{array}$ & $\begin{array}{c}70(47.4- \\
131.6)\end{array}$ & $\begin{array}{c}100(60- \\
100)\end{array}$ & $\begin{array}{c}100(55.8- \\
100)\end{array}$ & $0.057 *$ \\
\hline T stage & & & & & $0.941 * *$ \\
\hline $\mathrm{T}_{3}$ & 19 & 20 & 20 & 18 & \\
\hline $\mathrm{T}_{4}$ & 11 & 10 & 10 & 12 & \\
\hline $\mathrm{N}$ stage & & & & & $0.627 * *$ \\
\hline $\mathrm{N}_{0}$ & 10 & 15 & 12 & 12 & \\
\hline$\geqslant \mathrm{N}_{1}$ & 20 & 15 & 18 & 18 & \\
\hline M stage & & & & & $0.852 * *$ \\
\hline$M_{0}$ & 8 & 10 & 7 & 8 & \\
\hline$\geqslant \mathrm{M}_{1}$ & 22 & 20 & 23 & 22 & \\
\hline JW stage & & & & & $0.697 * *$ \\
\hline C & 2 & 5 & 4 & 4 & \\
\hline $\mathrm{D}$ & 28 & 25 & 26 & 26 & \\
\hline Gleason score & & & & & $0.968 * *$ \\
\hline$<7$ & 6 & 6 & 7 & 6 & \\
\hline 7 & 9 & 10 & 6 & 8 & \\
\hline$>7$ & 15 & 14 & 14 & 16 & \\
\hline
\end{tabular}

Abbreviations: CRT, conventional-dose beam radiotherapy; HIFU, high-intensity focused ultrasound; LRT, low-dose external beam radiotherapy; PSA, prostatespecific antigen. Age and initial PSA were showed as median and interquartile range. *Analysis of variance.

**Kruskal-Wallis test.

water was used as the medium between the concave spherical HIFU transducer and the patient's perineal region. The computer control station captured the prostate image through the ultrasound imaging transducer, selected the target region and then designed a treatment plan. According to the size of the prostate, the treatment region was divided into several layers from the base to the apex. Under the control of the computer program and real-time ultrasound monitoring, the focus was sequentially moved spot by spot in each layer, moving layer by layer from the base to the apex; the entire treatment was finished using an overlapping spot accumulation technique. The target region included the whole prostate and the proximal part of the seminal vesicle. The distance between the rectal mucosa and the dorsal prostate

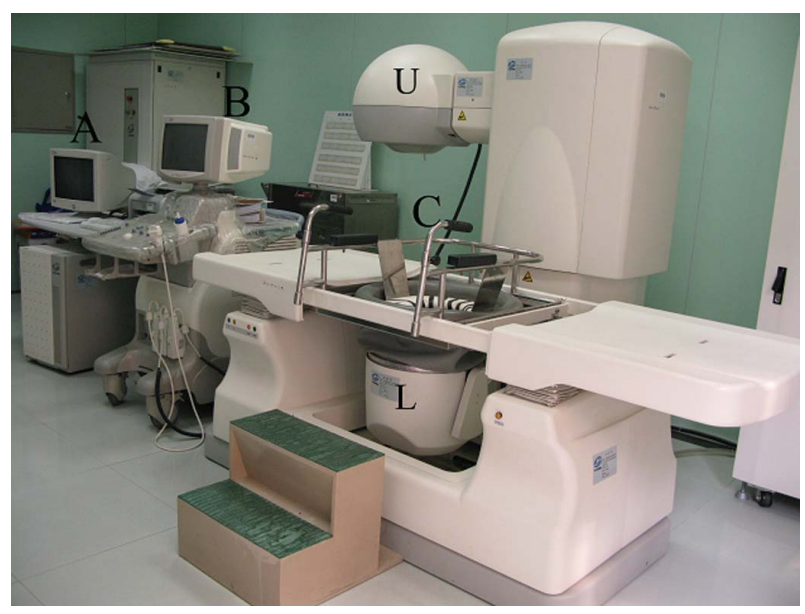

Figure 1 FEP-BY high-intensity focused ultrasound (HIFU) therapy system. (A) Computer control station; (B) ultrasound imaging system; (C) treatment table and seat frames; (U, L) upper and lower transducers. In addition, there is an electrical power system and water degassing system (no label). 
capsule ranged from 3 to $6 \mathrm{~mm}$. All HIFU treatments were applied by the same doctor.

\section{Radiotherapy}

The Saturne 41F 6MV photon linear accelerator (GE, Paris, France) was used for radiotherapy. The Oncor linear accelerator system (Siemens, Munich, Germany) was used from December 2005 until the end of the study. The CRT first delivers 'box' radiation doses of $40 \mathrm{~Gy}$ to the area of the pelvic lymph nodes and then delivers a reduced field boost of 25-30 Gy to the prostate and seminal vesicles, resulting in the accumulation of a total dose of over 65-70 Gy to the prostate area to ensure that treatment is effective.

\section{HIFU+LRT}

Compared with CRT, the new protocol for HIFU + LRT utilized HIFU in the treatment of primary tumour instead of the reduced field irradiation for the prostate and seminal vesicles, preserving only the dose of irradiation to the pelvic lymph node area. Therefore, the total dose of radiotherapy was reduced from $65-70$ to 40 Gy. All patients who underwent HIFU+LRT had an interval of about 1-2 weeks between the two treatments.

\section{Follow-up and statistical methodology}

Serum PSA was measured once a month up to normal level or a nadir. Thereafter, a serum PSA test and digital rectal examination were performed every 3 months. The data for all patients were added to our PCa database. A CT or MRI scan of the pelvis was obtained every 3-6 months. The overall survival (OS) and disease-specific survival (DSS) curves were calculated using the Kaplan-Meier method and compared by the log-rank test. Three end points were used in our data analysis. Time of death (or the last date of contact) was defined as the time from the date of diagnosis with PCa to the date of death (or to the last date of contact). In calculating the OS, all deaths were counted, regardless of the cause. Whereas the DSS was defined as the time of death from PCa itself, death from causes other than PCa and loss to follow-up were counted as terminal events. Multivariate survival analysis using Cox's regression model further assessed the effect of each treatment on survival to separate baseline hazard from other factors (such as age, initial PSA, Gleason score and stage). Meanwhile, the incidence and severity of the treatment-related GI and GU complications were recorded at each follow-up visit. Late radiation toxicity was scored according to the criteria of the Radiation Therapy Oncology Group/European Organization for Research and Treatment of Cancer. ${ }^{4}$ The morbidities of complications were compared using Fisher's exact probabilities. The primary end point of the study was overall survival. Secondary end points included disease-specific survival and late GU or GI toxicity. The statistical analyses were performed using Stata statistical software (version 9.0; StataCorp., College Station, TX, USA). $P<0.05$ was considered statistically significant. Medians (and interquartile ranges) were used for all descriptive statistics.

\section{RESULTS}

The median follow-up time (and interquartile ranges) of the control group, HIFU+LRT group, HIFU group and CRT group were 28.8 (17.8-51.7), 30.6 (7.4-52.7), 14.1 (8.2-28.7) and 55.1 (27.3-64.0) months, respectively. In the HIFU+LRT group, the median PSA nadir achieved was $0.10(0.05-1.12) \mathrm{ng} \mathrm{ml}^{-1}$ within $3.70(3.05-8.20)$ months. The CT/MR imaging scan showed that the primary tumours and pelvic lymph node metastases visibly shrank or even disappeared at 3-6 months after treatment in some cases (Figures 2 and 3).
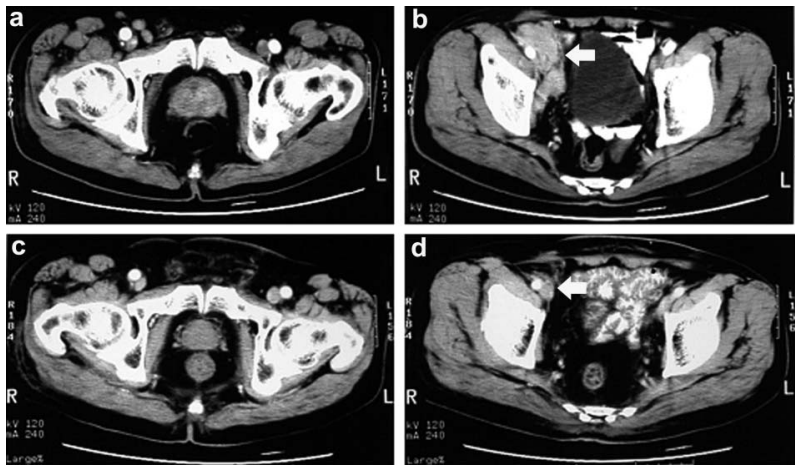

Figure 2 Case 1. Pre-treatment CT scans showed (a) enlargement of the prostate with irregular contrast enhancement and (b) right pelvic lymph node metastases around the iliac vessels (arrow). Six months later, post-treatment CT scans showed (c) significant prostate shrinkage with the absence of contrast enhancement and (d) disappearance of pelvic lymph node metastases (arrow).

We compared the long-term survival of the HIFU+LRT group with those of the other three groups. Figure 4 shows the Kaplan-Meier OS curves. Table 2 lists the 1-, 3- and 5-year OS rates. The long-term overall survival of the HIFU+LRT group was higher than those of the other three groups. The log-rank test suggested that OS values differed significantly $(P=0.018)$ among the four groups. Further comparisons were made between each pair of groups, and the Bonferroni correction was performed to test each of the individual tests at a significance level of $0.008(\alpha / 6)$. The results suggested that a significant difference existed between the HIFU+LRT group and the control group $(P=0.002)$, and no statistically significant difference was detected between any other two groups. Figure $\mathbf{5}$ showed the Kaplan-Meier DSS curves. Table 3 lists the 1-, 3- and 5-year DSS rates. The long-term disease-specific survival of the HIFU + LRT group was higher than those of the other three groups. The log-rank test suggested that DSS values differed significantly $(P=0.015)$ among the
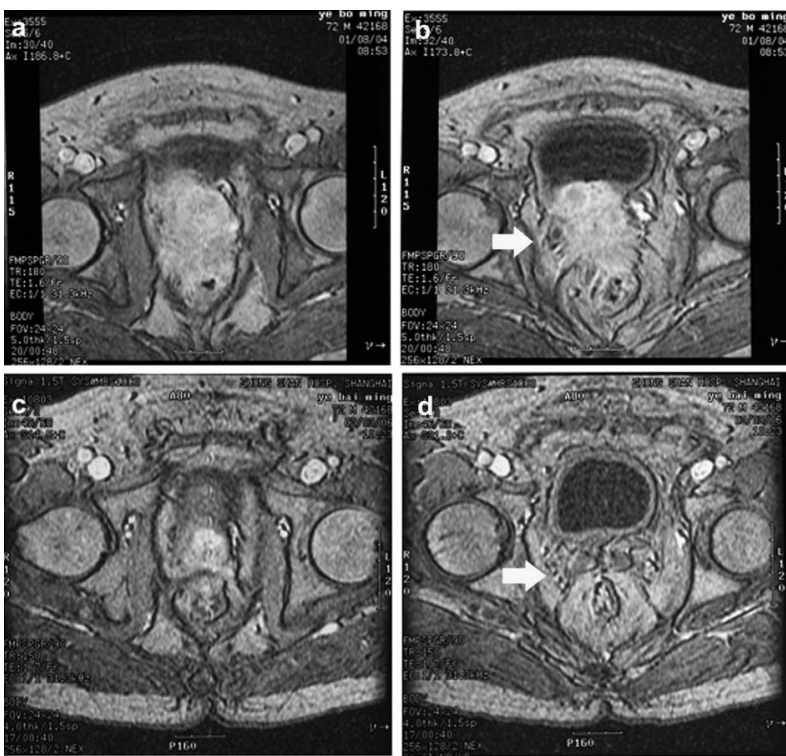

Figure 3 Case 2. Pre-treatment MR scans showed (a) tumor extended through the prostate capsule and (b) invaded seminal vesicles and adjacent structures including bladder neck, and rectum (arrow). Six months later, post-treatment MR scans showed (c) nearly complete tumor regression and (d) recovery of normal pelvic structures, such as seminal vesicles, bladder neck and rectum (arrow) 


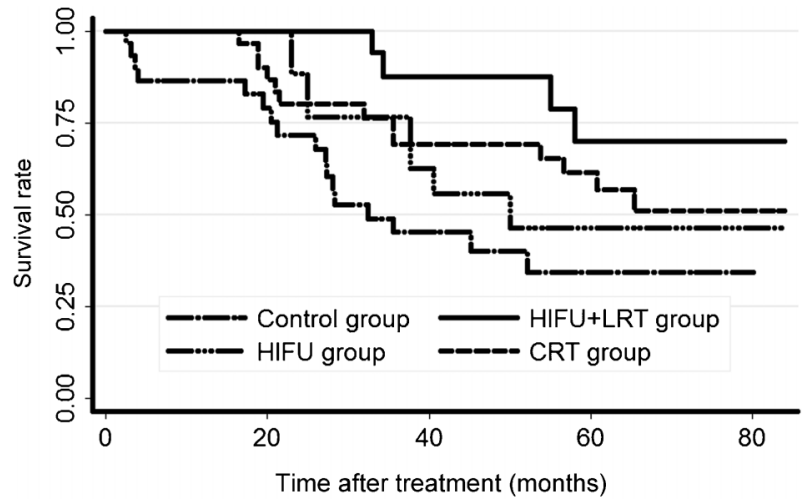

Figure 4 The overall survival curves. CRT, conventional-dose external beam radiotherapy; HIFU, high-intensity focused ultrasound; LRT, low-dose external beam radiotherapy.

Table 2 The 1-, 3- and 5-year OS rates ( $n=30$ in each group)

\begin{tabular}{lccc}
\hline \multirow{3}{*}{ Group } & \multicolumn{3}{c}{ OS rate (\%) } \\
\cline { 2 - 4 } & 1 year & 3 years & 5 years \\
\hline HIFU+LRT & 100.00 & 87.39 & 69.92 \\
HIFU & 100.00 & 62.57 & 46.35 \\
CRT & 96.67 & 69.09 & 56.69 \\
Control & 82.86 & 45.19 & 34.43 \\
\hline
\end{tabular}

Abbreviations: CRT, conventional-dose external beam radiotherapy; HIFU, highintensity focused ultrasound; LRT, low-dose external beam radiotherapy; OS, overall survival.

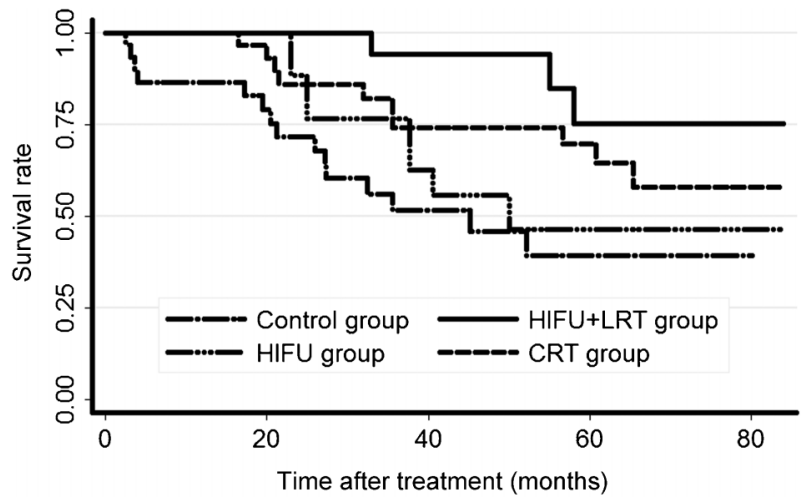

Figure 5 The disease-specific survival curves. CRT, conventional-dose external beam radiotherapy; HIFU, high-intensity focused ultrasound; LRT, low-dose external beam radiotherapy.

Table 3 The 1-, 3- and 5-year DSS rates ( $n=30$ in each group)

\begin{tabular}{lccc}
\hline \multirow{3}{*}{ Group } & \multicolumn{3}{c}{ DSS rate (\%) } \\
\cline { 2 - 4 } & 1 year & 3 years & 5 years \\
\hline HIFU+LRT & 100.00 & 94.12 & 75.29 \\
HIFU & 100.00 & 62.57 & 46.35 \\
CRT & 96.67 & 74.12 & 64.47 \\
Control & 82.86 & 51.65 & 39.35 \\
\hline
\end{tabular}

Abbreviations: CRT, conventional-dose external beam radiotherapy; DSS, diseasespecific survival; HIFU, high-intensity focused ultrasound; LRT, low-dose external beam radiotherapy.
Table 4 Multivariate models predicting overall survival

\begin{tabular}{lll}
\hline Variable & Hazard ratio $(95 \% \mathrm{Cl})$ & $\mathrm{P}$ \\
\hline HIFU+LRT & $0.108(0.028-0.413)$ & 0.001 \\
HIFU & $0.648(0.257-1.633)$ & 0.358 \\
CRT & $0.459(0.192-1.097)$ & 0.080 \\
Age & $1.867(0.818-4.260)$ & 0.138 \\
Initial PSA & $0.504(0.240-1.059)$ & 0.070 \\
Gleason score & $3.617(1.246-10.502)$ & 0.018 \\
T stage & $1.813(0.938-3.504)$ & 0.077 \\
N stage & $4.235(1.616-11.095)$ & 0.003 \\
M stage & $4.462(1.515-13.142)$ & 0.007 \\
\hline
\end{tabular}

Abbreviations: $\mathrm{Cl}$, confidence interval; $\mathrm{CRT}$, conventional-dose external beam radiotherapy; HIFU, high-intensity focused ultrasound; LRT, low-dose external beam radiotherapy; PSA, prostate-specific antigen.

four groups, but further comparison between each pair of groups suggested that a significant difference existed between the HIFU+LRT group and control group $(P=0.003)$, whereas no statistically significant difference was detected between any other two groups.

Multivariable Cox's proportional hazard model indicated that Gleason score, $\mathrm{N}$ stage, $\mathrm{M}$ stage and treatment modalities were important predictors of survival in advanced PCa, whereas age, initial PSA and $\mathrm{T}$ stage were not (Tables 4 and 5). For HT followed by HIFU+LRT (or CRT) versus HT alone, the former had a protective effect on the hazard of death. HIFU+LRT was significantly associated with both OS $(P=0.001)$ and DSS $(P=0.001)$, and CRT was significantly associated with DSS $(P=0.035)$. HT followed by HIFU alone had no significant protective effect on the hazard of death.

To compare the morbidities of severe late GI and GU complications $\geqslant$ grade II among patients who underwent HIFU and received varying doses of radiotherapy, we analysed the long-term follow-up results of the following three groups: the HIFU group, the HIFU+LRT group and the CRT group. Late ( $>120$ days) radiation toxicity was evaluated and scored according to the criteria published by the Radiation Therapy Oncology Group/European Organization for Research and Treatment of Cancer. ${ }^{4}$

All patients were divided into two parts according to whether they experienced severe late toxicity grade $\geqslant$ II (Table 6). In the HIFU group and the HIFU+LRT group, only seven patients had transient slight haematuria requiring no medication during the first 2 months after HIFU treatment; no patients had late GU complications. In the CRT group, three patients had recurrent gross haematuria requiring medication; two patients had long-term bladder irritation and frequency of urination 2 times/h during the day; three patients had recurrent gross

Table 5 Multivariate models predicting disease-specific survival

\begin{tabular}{lll}
\hline Variable & Hazard ratio $(95 \% \mathrm{Cl})$ & $\mathrm{P}$ \\
\hline HIFU+LRT & $0.080(0.017-0.364)$ & 0.001 \\
HIFU & $0.675(0.258-1.763)$ & 0.422 \\
CRT & $0.347(0.129-0.930)$ & 0.035 \\
Age & $1.409(0.576-3.445)$ & 0.452 \\
Initial PSA & $0.458(0.201-1.043)$ & 0.063 \\
Gleason score & $3.164(1.043-9.595)$ & 0.042 \\
T stage & $1.914(0.939-3.903)$ & 0.074 \\
N stage & $3.778(1.297-11.001)$ & 0.015 \\
M stage & $5.488(1.596-18.873)$ & 0.007 \\
\hline
\end{tabular}

Abbreviations: $\mathrm{Cl}$, confidence interval; $\mathrm{CRT}$, conventional-dose external beam radiotherapy; HIFU, high-intensity focused ultrasound; LRT, low-dose external beam radiotherapy; PSA, prostate-specific antigen. 
Table 6 The morbidities of late GU and GI complications ( $n=30$ in each group)

\begin{tabular}{|c|c|c|c|c|}
\hline \multirow{2}{*}{ Group } & \multicolumn{2}{|c|}{ GU complications } & \multicolumn{2}{|c|}{ Gl complications } \\
\hline & No or grade I & $\geqslant$ grade /I & No or grade I & $\geqslant$ grade II \\
\hline $\operatorname{HIFU}(n)$ & 30 & 0 & 30 & 0 \\
\hline HIFU+LRT (n) & 30 & 0 & 30 & 0 \\
\hline CRT (n) & 22 & 8* & 20 & $10 * *$ \\
\hline
\end{tabular}

Abbreviations: CRT, conventional-dose external beam radiotherapy; GI, gastrointestinal; GU, genitourinary; HIFU, high-intensity focused ultrasound; LRT, low-dose external beam radiotherapy.

$* P<0.0001$, compared with HIFU group and HIFU+LRT group.

$* * P<0.0001$, compared with HIFU group and HIFU+LRT group.

haematuria and urinary retention requiring medication and permanent (or suprapubic) catheter. In the HIFU group and the HIFU+LRT group, no patient had severe late GI complications. In the CRT group, 10 patients had long-term recurrent rectal irritation requiring medication, such as moderate diarrhoea or intermittent severe cramping. The statistic test results of Fisher's exact probabilities indicated significant differences in the morbidity of grade II or higher GU and GI complications between CRT group and other two groups. No severe GU or GI complications were found in the HIFU group or the HIFU+LRT group; therefore, the results suggested that conventional-dose radiotherapy induced higher morbidity of severe GU and GI complications.

\section{DISCUSSION}

Currently, the preferred primary treatment for advanced $\mathrm{PCa}$ is androgen deprivation therapy (ADT), conducted by the principle of intermittent maximal androgen blockade. The guidelines of the Chinese Urological Association use a single threshold for stopping or resuming $\mathrm{ADT}^{9}$ in all prostate cancers, but the majority of literatures, including those proposed by the European Association of Urology guidelines, ${ }^{10}$ adopted a higher threshold in advanced or metastatic disease than in relapsing disease after radical therapy for prostate cancer. ${ }^{11-13}$ At present, there is no consensus with regard to the optimal threshold. The most commonly used PSA value for the withdrawal of ADT is less than $4 \mathrm{ng} \mathrm{ml}^{-1}$, and the most widely used PSA value for the resumption of ADT is more than 10 or $20 \mathrm{ng} \mathrm{ml}^{-1}$. $^{11-13}$ Most patients with advanced PCa were able to achieve temporary palliation at the beginning of $\mathrm{HT}$, and intermittent maximal androgen blockade may help delay progression to androgen independence; unfortunately, however, nearly all hormone-sensitive PCa patients ultimately develop hormone-refractory PCa within a median of 18 24 months. $^{2}$ At present, there are no effective treatment options, and most patients have a very poor prognosis with a subsequent median survival time of no more than 2 years. ${ }^{14}$ Adjuvant radiotherapy may improve local control for advanced $\mathrm{PCa}$, and delivering a higher radiation dose may improve efficacy. ${ }^{15}$ However, an increase of the radiation dose to the tumour implies an increase of the dose to the surrounding normal tissue, which results in elevated incidence of severe late GU and GI complications (such as radiocystitis, radiation colitis and, especially, proctitis). ${ }^{4,5}$ In recent years, increasing attention has been paid to the use of combination therapy for advanced cancer. Currently, a few novel, minimally invasive techniques have been applied in the treatment of solid tumours, and the focus is on whether these minimally invasive therapies can replace or modify the traditional radiotherapy procedure to involve a safe, reduced radiation dose.

HIFU is a very attractive option. In 1995, Madersbacher et al. ${ }^{16}$ first reported the clinical application of HIFU to treat localized PCa. Since then, many studies have confirmed the efficacy and safety of HIFU in the treatment of early localized PCa. ${ }^{17,18}$ However, clinical results have shown a substantially increased ratio of biochemical recurrence and positive biopsy among the patients with high-risk or locally advanced PCa who underwent HIFU therapy. ${ }^{19,20}$ This may have resulted from the following factors: (i) the ultrasound positioning system could not accurately demarcate the invasive margin of advanced PCa, which led to the insufficiency of treatment; and (ii) patients with high-risk or locally advanced PCa had a high risk of pelvic lymph node involvement due to the blind zone beyond target areas of HIFU treatment. These patients had a high PSA value or Gleason score. According to the formula for predicting the risk of positive lymph nodes $\left(\mathrm{N}_{+}\right)$proposed by Roach et al., ${ }^{21} \mathrm{~N}_{+}=2 / 3$ (PSA) $+(\mathrm{GS}-6) \times 10$ (where GS is Gleason score), even if no evidence of metastasis was detected by the imaging examination, the patients probably still had occult pelvic lymph node spread. Thus, efficacy of HIFU treatment was poor as a solo treatment for advanced PCa. Our study suggests that OS and DSS did not differ significantly between the HIFU group and control group, which also confirms the above-described hypothesis.

For the above reasons, we devised a combined treatment protocol involving HIFU and LRT for advanced PCa. Currently, radiotherapy might be the most effective treatment for pelvic metastases. Radiotherapy was able to cover the blind zone of HIFU treatment (i.e., the invasive margin of the tumour and pelvic lymph nodes). In turn, HIFU could accurately induce coagulative necrosis within the prostatic target area without damaging surrounding normal tissues, which could compensate for the shortage of radiotherapy to the primary tumour. Therefore, the two treatments were complementary. In addition, there was a synergistic effect between HIFU and radiotherapy, which could further enhance the effect on primary tumours. In addition to the reliable local therapeutic effects on primary tumour, some evidence indicated that the remaining tumour debris after HIFU ablation released tumour antigens that induced a systemic antitumour immune response. This systemic effect might facilitate control of the primary tumour and metastatic lesions. ${ }^{22,23}$ Our study found that as supplemental therapy after HT for advanced PCa, HIFU+LRT effectively inhibited the primary tumours and pelvic lymph node metastases, prolonging the survival time of patients. Compared with HT alone, supplemental HIFU + LRT or CRT had protective effects on the risk of death, but HIFU alone did not. The long-term diseasespecific survival of the HIFU+LRT group was significantly higher than that of the group treated with HT alone, but differences between the HIFU+LRT group and the CRT group had no statistical significance. Notably, the results cannot exclude the possibility of a falsenegative due to the small sample size used. Based on the research outlined above, we conclude that strengthening local control of primary tumour and regional lymph node metastases were essential to advanced PCa after HT and that the efficacy of HIFU+LRT was greater than that of HIFU alone and not inferior to CRT.

Because prostate cancer patients have a potentially long period of survival, assessment of late treatment-related toxicity is important. The data collected showed that the GI and GU toxicity grade II or higher caused by CRT was 23.2 and $28.5 \%$, respectively. ${ }^{4}$ The complications of HIFU were less, only a few patients complained of acute urinary retention, mild urinary incontinence and so on, but severe complications such as rectourethral fistula and rectal mucosa burning were rare. Moreover, with the improvement of HIFU procedures and devices, the incidence of severe complications has been reduced to almost zero in recent studies. ${ }^{24}$ Blana et al. ${ }^{25}$ reported that the incidence of severe complications was still low in those patients who 
underwent repeated HIFU treatment due to progression of the disease. We devised the combined treatment protocols, applying HIFU for the treatment of prostatic primary tumour to replace box irradiation to prostate and seminal vesicles in CRT while preserving the dose of irradiation to the pelvic lymph node area. Based on the good local control of HIFU, as well as the complementation and synergism with radiotherapy, we had every reason to reduce the dose of irradiation to the prostate so as to reduce the influence of radiation on the GI and GU tract around the prostate. Our study showed that no late GI or GU complication of grade II or higher occurred in any case that underwent HIFU alone or HIFU + LRT; however, these complications were common among patients who underwent CRT. Therefore, compared with CRT, HIFU+LRT significantly decreased the incidence of severe radiation-related late GU and GI complications.

In conclusion, for patients with advanced $\mathrm{PCa}$, long-term survival benefited from strengthening local control of the primary tumour and regional lymph node metastases after HT. As an alternative to CRT, HIFU+LRT showed good efficacy and better safety.

\section{AUTHOR CONTRIBUTIONS}

RYW designed the study, performed experiments, analysed data and wrote the paper; GMW designed and supervised the study, and edited the manuscript; LX performed treatments and follow-up of all patients, analysed data and wrote the paper; BHZ supervised its analysis and edited the manuscript; YQX performed treatment and follow-up of HIFU; ZCZ gave technical support and conceptual advice; $\mathrm{BC}$ performed treatment and follow-up of radiotherapy.

\section{COMPETING FINANCIAL INTERESTS}

The authors declare no competing financial interests.

\section{ACKNOWLEDGMENTS}

This work was supported by the fund of the Health Bureau of Shanghai Municipality, China (No. 2006016).

1 Sim HG, Cheng CW. Changing demography of prostate cancer in Asia. Eur J Cancer 2005; 41: 834-45.

2 Chang SS, Kibel AS. The role of systemic cytotoxic therapy for prostate cancer. BJU Int 2009; 103: 8-17.

3 Bubendorf L, Schöpfer A, Wagner U, Sauter G, Moch H et al. Metastatic patterns of prostate cancer: an autopsy study of 1,589 patients. Hum Pathol 2000; 31: 578-83.

4 Peeters ST, Heemsbergen WD, van Putten WL, Slot A, Tabak H et al. Acute and late complications after radiotherapy for prostate cancer: results of a multicenter randomized trial comparing 68 Gy to 78 Gy. Int J Radiat Oncol Biol Phys 2005; 61 : 1019-34.
5 Zietman AL, Coen JJ, Dallow KC, Shipley WU. The treatment of prostate cancer by conventional radiation therapy: an analysis of long-term outcome. Int J Radiat Oncol Biol Phys 1995; 32: 287-92.

6 Kennedy JE. High-intensity focused ultrasound in the treatment of solid tumours. Nat Rev Cancer 2005; 5: 321-7.

7 Jernberg A, Edgren MR, Lewensohn R, Wiksell H, Brahme A. Cellular effects of highintensity focused continuous wave ultrasound alone and in combination with $\mathrm{X}$-rays. Int J Radiat Biol 2001; 77: 127-35.

8 Jun-Qun Z, Guo-Min W, Bo Y, Gong-Xian W, Shen-Xu H. Short-term results of 89 cases of rectal carcinoma treated with high-intensity focused ultrasound and low-dose radiotherapy. Ultrasound Med Biol 2004; 30: 57-60.

9 Li M. Guidelines on diagnosis and treatment of prostate cancer. In: Na YQ, Sun G, editors. Chinese Guidelines on Diagnosis and Treatment of Urological Disease. Beijing: People's Medical Publishing House; 2009. p47.

10 Heidenreich A, Bolla M, Joniau S, van der Kwast TH, Matveev V et al. Guidelines on Prostate Cancer (Online). Arnhem: European Association of Urology; 2010. p91.

11 Theyer G, Hamilton G. Current status of intermittent androgen suppression in the treatment of prostate cancer. Urology 1998; 52: 353-9.

12 Salonen AJ, Viitanen J, Lundstedt S, Ala-Opas M, Taari K et al. Finnish multicenter study comparing intermittent to continuous androgen deprivation for advanced prostate cancer: interim analysis of prognostic markers affecting initial response to androgen deprivation. J Urol 2008; 180: 915-9.

13 Abrahamsson PA. Potential benefits of intermittent androgen suppression therapy in the treatment of prostate cancer: a systematic review of the literature. Eur Urol 2010; 57: 49-59.

14 Cheville JC, Tindall D, Boelter C, Jenkins R, Lohse CM et al. Metastatic prostate carcinoma to bone: clinical and pathologic features associated with cancer-specific survival. Cancer 2002; 95: 1028-36.

15 Pollack A, Smith LG, von Eschenbach AC. External beam radiotherapy dose response characteristics of 1127 men with prostate cancer in the PSA era. Int J Radiat Oncol Biol Phys 2000; 48: 507-12.

16 Madersbacher S, Pedevilla M, Vingers L, Susani M, Marberger M. Effect of highintensity focused ultrasound on human prostate cancer in vivo. Cancer Res 1995; 55: 3346-51.

17 Chaussy $C$, Thüroff S. Results and side effects of high-intensity focused ultrasound in localized prostate cancer. J Endourol 2001; 15: 437-40.

18 Uchida T, Sanghvi NT, Gardner TA, Koch MO, Ishii D et al. Transrectal high-intensity focused ultrasound for treatment of patients with stage T1b-2NOMO localized prostate cancer: a preliminary report. Urology 2002; 59: 394-9.

19 Ficarra V, Antoniolli SZ, Novara G, Parisi A, Fracalanza S et al. Short-term outcome after high-intensity focused ultrasound in the treatment of patients with high-risk prostate cancer. BJU Int 2006; 98: 1193-8.

20 Kiel HJ, Wieland WF, Rössler W. Local control of prostate cancer by transrectal HIFUtherapy. Arch Ital Urol Androl 2000; 72: 313-9.

21 Roach M 3rd, Marquez C, Yuo HS, Narayan P, Coleman L et al. Predicting the risk of lymph node involvement using the pre-treatment prostate specific antigen and Gleason score in men with clinically localized prostate cancer. Int J Radiat Oncol Biol Phys 1994; 28: 33-7.

22 Hu Z, Yang XY, Liu Y, Morse MA, Lyerly HK et al. Release of endogenous danger signals from HIFU-treated tumor cells and their stimulatory effects on APCs. Biochem Biophys Res Commun 2005; 335: 124-31.

23 Rosberger DF, Coleman DJ, Silverman R, Woods S, Rondeau M et al. Immunomodulation in choroidal melanoma: reversal of inverted CD4/CD8 ratios following treatment with ultrasonic hyperthermia. Biotechnol Ther 1994; 5: 59-68.

24 Chaussy $C$, Thüroff $S$. High intensity focused ultrasound in prostate cancer: results after 3 years. Mol Urol 2000; 4: 179-82.

25 Blana A, Rogenhofer S, Ganzer R, Wild PJ, Wieland WF et al. Morbidity associated with repeated transrectal high-intensity focused ultrasound treatment of localized prostate cancer. World J Urol 2006; 24: 585-90. 